\title{
Analysis of Solid-Liquid Two-Phase Flow Field for Shellfish Precooking Processing Machine
}

\author{
Xindan Zhang ${ }^{1,2}$, Xu Zhang ${ }^{1,2}$, Lianzheng Sun ${ }^{1,2}$, Jinshi Lu ${ }^{1,2}$, Xueheng Tao ${ }^{1,2, ~ * ~}$ \\ ${ }^{1}$ Key Laboratory for Seafood Processing Technology and Equipment of Liaoning Province, Dalian Polytechnic University, Dalian, China \\ ${ }^{2}$ National Engineering Research Center of Seafood, Dalian, China
}

Email address:

xhtao@dlpu.edu.cn (Xueheng Tao)

${ }^{*}$ Corresponding author

\section{To cite this article:}

Xindan Zhang, Xu Zhang, Lianzheng Sun, Jinshi Lu, Xueheng Tao. Analysis of Solid-Liquid Two-Phase Flow Field for Shellfish Precooking Processing Machine. International Journal of Mechanical Engineering and Applications. Vol. 5, No. 1, 2017, pp. 20-27. doi: 10.11648/j.ijmea.20170501.13

Received: January 5, 2017; Accepted: January 13, 2017; Published: February 10, 2017

\begin{abstract}
A new type of shellfish precooking processing machine is designed. The corresponding three-dimensional solid model are constructed by use of SOLWORKS. It also introduces basic structure and working principle of shellfish precooking machine. Solid-liquid two-phase flow field for shellfish precooking processing machine is simulated by ANSYS FLUENT 15.0, which simulated the distribution of liquid flow and volume fraction of solid in the pot. The reasons of flow field distribution and the solid particle volume fraction of the liquid phase flow in the vertical and horizontal planes were analyzed under different mixing speeds. The results show that the solid-liquid two-phase flow field distribution is reasonable and turbulent region can be formed in the pot. The distribution of flow field and the texture properties of abalone were compared at three different speeds, and the more reasonable mixing speed was determined. The results of the analysis provide references for the optimization of mixer in the future.
\end{abstract}

Keywords: Shellfish Precooking Processing Machine, Mixer, Solid-Liquid Two-Phase Flow Field, Analysis

\section{Introduction}

Marine products has been an important human life resources since ancient times. The coastal residents in ancient China have been working on the development and utilization of marine products. Especially the development of shellfish put a great effort [1]. The processing of shellfish has a long history. But development has been in the initial stage. There are a series of problems, such as obsolete machine, high energy consumption and low degree of automation, which needs to be solved urgently [2]. In the traditional processing products, shellfish resource utilization is low, there is no better development and utilization of waste, low level of mechanization and processing, product quality is not high [3].

With the continuous increase of shellfish production and rising labor costs, more and more processing enterprises are aware of the importance of processing equipment and gradually increase the application and investment of processing equipment in recent years. Currently, the application rate of shellfish processing equipment has increased year by year in Jiangsu, Shandong, Liaoning, Guangdong, Zhejiang and other shellfish processing industry in the more developed regions [4]. Live in fresh, pre-treatment, primary processing, deep processing and comprehensive utilization of a plurality of links are using the appropriate machine to improve processing efficiency. Foreign put great importance on processing of shellfish, the shellfish processing industry are more developed in Western Europe and other countries, shellfish in addition to fresh, but also be made into canned or fried, roasted, boiled and steamed, smoked and frozen food. Frozen processing is the largest in New Zealand and South Korea; the production of shellfish in the dry process of meat is used in Germany. Abalone and other valuable shellfish can be used to freeze by liquid quick-freezing machine [5]. The high pressure processing technology has gradually been used a number of companies at precooking shellfish in the USA [6]. Development and research of shellfish food processing machine have a very broad prospects. 
To meet market demand, for this series of questions, a new shellfish precooking processing machine is put forward combined with the advanced processing technology in this paper. The shellfish precooking machine makes use of controlled silicon to control temperature, which achieve multi-segment and multi-step temperature control, with less time and a small amount of temperature change to get processing technology. The irregular hemisphere tilting steam jacket kettle is applied to shellfish precooking processing machine for achieving high production efficiency. In order to be sure to conditioning fluid to generate turbulence, uniform heat perception of materials and reasonable tasty during the processing. The shape of the mixer is matched with the precooking device, which is tubular cross circular arc, so as to reduce the damage to the shellfish and achieve the purpose of mixer evenly. The fluid field in the mixer is simulated. The results show the solid-liquid two-phase flow field distribution is reasonable and the mixer is uniform.

\section{Materials and Methods}

\subsection{Basic Structure and Working Principle of Shellfish Precooking Machine}

The main structure of the shellfish precooking machine is shown in Figure 1, which consisted of the frame, the elevating device, the precooking device and the mixer. The elevating device and the precooking device are fixed on the stander. The elevating device is used to complete the pot cover and the vertical movement of the mixer.

The elevating device is mainly composed of the intelligent combination unit that completes the vertical work station of the conversion, whose advantage is higher operating speed, lower noise and longer maintenance-free.

The mixer consists of an electric motor, a mixer and cover. The motor and the mixer fixed to the cover. The mixer is installed on the elevating device, which moved vertically along it. The precooking device mainly consists of electric motor, worm gear reducer and steam sandwich pot.

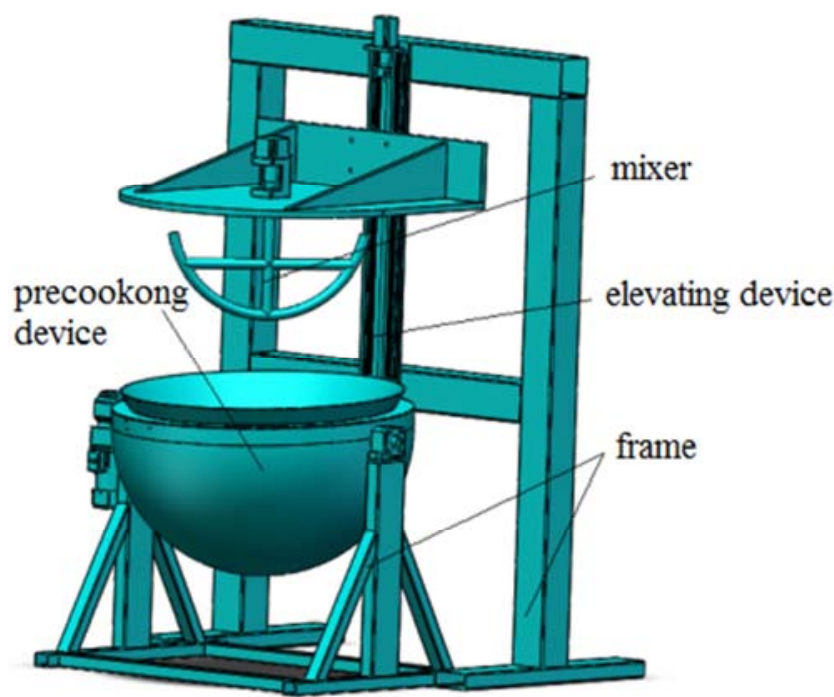

Figure 1. Structure of the shellfish precooking machine.
The precooking device is controlled to turn and unload automatically by the electric motor. The tilting steam jacket kettle has the characteristics of large heating area, high heat efficiency and uniform heating.

The working principle of precooking shellfish processing machine are described as follows. The intelligent combination unit rotary rises by the electric motor. Under the effect of the elevating device, the lid rises along with a mixer for placing the material in preparation. The material is put into the precooking steam jacket kettle by means of automatic feeding device. After that, the lid and the mixer return to the original workstation. The material is heated by steam, and then the precooking is started. The technological procedure is as showed in Figure 2 [7].

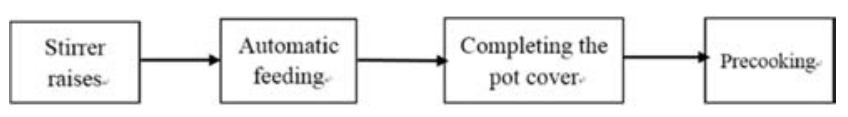

Figure 2. Technological procedure.

\subsection{Numerical Simulation of Flow in Mixing}

\subsubsection{Calculation Model}

Precooking material are shelled shellfish and regulation liquid, which is numerical simulation of solid-liquid two-phase flow in Stirred. ANSYS FLUENT 15.0 was used as the simulation tool. In the solid-liquid two-phase flow, the movement of solid particles is affected by two kinds of motion - the effects of random motion and follow the movement of fluid entrained, which mainly depend on the local fluid speed, the settling speed of the particles and the local turbulence intensity [8]

At present, the numerical simulation of two phase flow are mainly Lagrange and Euler [9]. Lagrange is to take the fluid as a continuous medium, and the particles are regarded as discrete system. The equations are used to simulate the flow field in the Euler coordinate system to solve the Reynolds equation of the fluid phase. The trajectory model of solid particles was obtained by the track model of the Lagrange coordinate system. It is assumed that the liquid phase affects the movement of the particles and is not affected by the movement of the particles. Particles are treated as fluid, it is considered that the particle and fluid are the continuous medium of mutual penetration and two phases in the Euler coordinate. The Euler equation and the RNG k- $\varepsilon$ turbulent model were used in this paper. The relevant equations of the model are expressed as [10-11]:

(1) Continuous equation:

$$
\frac{\partial}{\partial \mathrm{t}}\left(\alpha_{q}\right)+\nabla\left(\alpha_{q} v_{q}\right)=\frac{1}{P_{q}}\left(\sum_{p=0}^{n} \dot{m}_{p q} \alpha_{q} \frac{d \rho_{q}}{d t}\right)
$$

Where $\alpha_{q}$ is the volume fraction of phase $\mathrm{q}, v_{q}$ is the speed of phase q, $\dot{m}_{p q}$ is mass transfer from phase q to phase $\mathrm{p}, p_{q}$ is the pressure of phase $\mathrm{q}, \rho_{q}$ is the density of phase $\mathrm{q}$.

(2) Momentum equation:

Liquid phase momentum equation:

$$
\frac{\partial}{\partial t}\left(\alpha_{l} p_{l} v_{l}\right)+\nabla\left(\alpha_{l} p_{l} v_{l i} v_{l j}\right)=-\alpha_{l} \nabla p+\nabla \overline{\overline{\bar{\tau}_{l}}}
$$




$$
\begin{aligned}
& +\alpha_{l} p_{l} g+\alpha_{l} p_{l}\left(F_{l}+F_{l i f t, l}+F_{v m, l}\right) \\
& +\sum_{i=1}^{n}\left[K_{l s}\left(v_{l}-v_{s}\right)+\dot{m}_{l s}\right]
\end{aligned}
$$

Solid phase momentum equation:

$$
\begin{gathered}
\frac{\partial}{\partial t}\left(\alpha_{s} p_{s} v_{s}\right)+\nabla\left(\alpha_{s} p_{s} v_{s i} v_{s j}\right)=-\alpha_{s} \nabla p+\nabla \overline{\bar{\tau}_{s}} \\
+\alpha_{s} p_{s} g+\alpha_{l} p_{s}\left(F_{s}+F_{l i f t, s}+F_{v m, s}\right) \\
+\sum_{i=1}^{n}\left[K_{l s}\left(v_{l}-v_{s}\right)+\dot{m}_{l s}\right]
\end{gathered}
$$

Where $k_{l s}$ is exchange coefficient of momentum between the liquid phase and the solid phase, $F$ is external force, $F_{\text {lift }}$ is lift force; $\overline{\bar{\tau}}$ is shear tensile stress.

(3) Turbulent kinetic energy equation:

$$
\rho \frac{D k}{D x_{i}}=\frac{\partial}{\partial x_{i}}\left(\alpha_{k} \mu_{f f} \frac{\partial k}{\partial x_{i}}\right)+G_{k}-\rho \varepsilon
$$

(4) Turbulent energy dissipation rate equation:

$$
\rho \frac{D \varepsilon}{D t}=\frac{\partial}{\partial x_{i}}\left(\alpha_{\varepsilon} \mu_{f f} \frac{\partial \varepsilon}{\partial x_{i}}\right)+C_{1 \varepsilon} \frac{\varepsilon}{k} G_{k} C_{2 \varepsilon} \rho \frac{\epsilon^{2}}{k}-R
$$

The correction factor $R$ is calculated as follows:

$$
R=\frac{C_{\mu} \rho \eta^{3}\left(1-\eta / \eta_{0}\right)}{1+\beta \eta^{3}} \frac{\varepsilon^{2}}{k}
$$

The turbulent viscosity $\mu_{t}$, the model parameter $\eta$ and the production of turbulent kinetic energy $G_{k}$ are calculated as follows:

$$
\begin{gathered}
G_{k}=-\rho \overline{\dot{u}_{c l} \dot{u}_{c j}} \frac{\partial u_{c j}}{\partial x_{i}} \\
\eta=\frac{k}{\varepsilon} \sqrt{\frac{G_{k}}{\mu_{t}}} \\
\mu_{t}=\rho C_{\mu} \frac{k^{2}}{\varepsilon}
\end{gathered}
$$

The adjustable constant $C_{1 \varepsilon}, C_{2 \varepsilon}, \eta_{0}, \beta$ and $C_{\mu}$ are obtained by sufficient data statistics [12]:

$$
\begin{gathered}
C_{1 \varepsilon}=1.42, C_{2 \varepsilon}=1.68, \eta_{0}=4.38, \\
\beta=0.012, C_{\mu}=0.09 .
\end{gathered}
$$

\subsubsection{Parameters of the Mixer and Material}

The pot body is an irregular hemisphere. The bottom of the pot is hemispherical, whose diameter is $700 \mathrm{~mm}$. The total height is $365 \mathrm{~mm}$. As shown in Figure 3, the shape of the mixer is matched with the precooking device, which is tubular cross circular arc. The diameter of the arc is $570 \mathrm{~mm}$. The diameter of the tubular section is $30 \mathrm{~mm}$. The distance between the center of a circle and cross horizontal tube is $150 \mathrm{~mm}$. And the distance of between the bottom of the pot and mixer is $60 \mathrm{~mm}$.

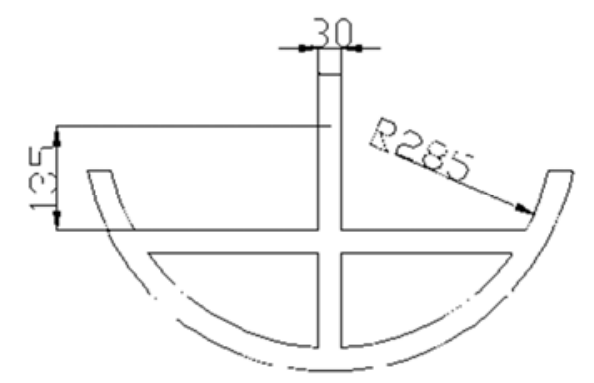

Figure 3. Schematic diagram of the structure of mixer.

The material to be stirred is solid liquid two phase entity of the flow. The density liquid phase is $1000 \mathrm{~kg} / \mathrm{m}^{3}$. The density of the solid phase is $1118 \mathrm{~kg} / \mathrm{m}^{3}$. And the diameter on the average is $36 \mathrm{~mm}$. According to the process characteristics of canned food, the ratio of material to water was determined as $1: 2$ [13]. The volume fraction is $33 \%$.

\subsubsection{Grid Partitioning}

In order to shorten the calculation time, the whole calculation area was simplified. The main body of the fluid domain was divided into grid by using tetrahedral structure. Figure 4 shows the final mesh of the model, in which (a) is global computational domain mesh model and (b) is mesh of the mixer. At the same time, the sliding grid method was adopted to divide the calculation area, which set up the dynamic and static regions [14]. The foundation of the sliding mesh model is to build an interchange interface at the turn/static interference, which makes the calculation domain to generate two relative motion sub domains that are connected by the non-uniformity grid interface. Therefore, in the division of the grid, it considered that the flow of fluid into the two sub domains of dynamic and static in different regions of the grid. Figure 5 shows the mesh model of static region. Figure 6 shows the mesh model of dynamic region. The total number of grid is 838,881 .

Mesh selection methods mentioned above to ensure that the grid in the model structure of the complex of the grid refinement and model in a single local grid is relatively sparse. At the same time, the grid change ratio is not too large. In this way, we can not only guarantee the quality of the grid, but also reduce the number of the grid, which can ensure the accuracy of the calculation and speed up the calculation.

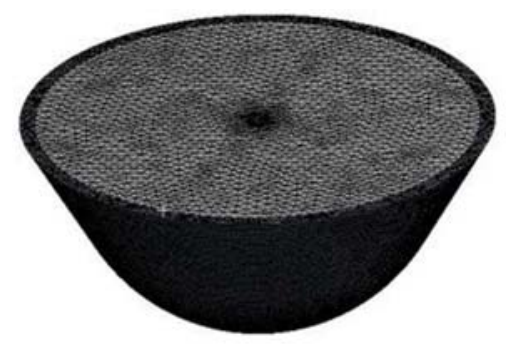

(a) 


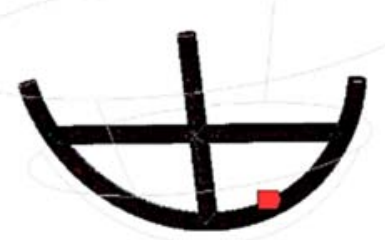

(b)

Figure 4. Global computational domain mesh model.

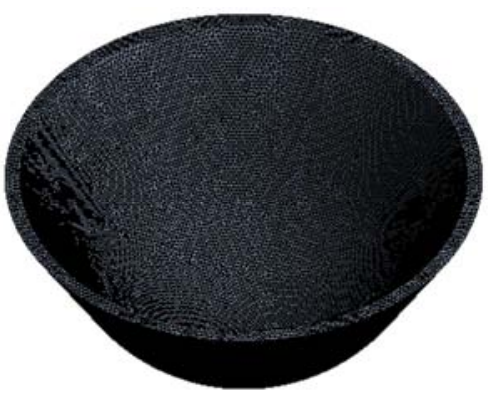

Figure 5. Mesh model of static region.

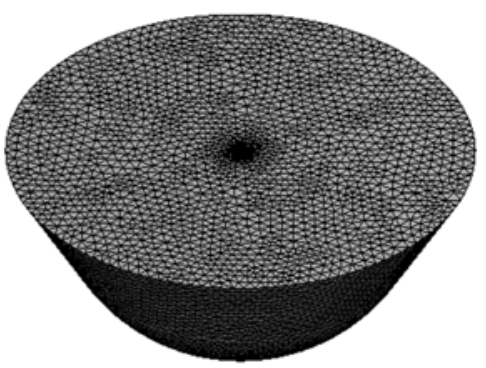

Figure 6. Mesh model of dynamic region.

\subsubsection{Setting of the Boundary Conditions}

The boundary conditions were set and the flow field was set to the closed flow field in the computational domain of grid. Considering the difference of two kinds of liquid density difference, the effect of gravity should be considered in simulation. The standard wall function was adopted in the area of the wall of the mixing tank. The wall was set to no slip boundary. The surface of the fluid region and the mixer paddle was arranged as a rotating wall surface. The constant temperature steam heating was used. The steam was filled with the sandwich layer of the pot, and temperature was $110^{\circ} \mathrm{C}$. According to the literature research [15], the speed of the mixer is set to $15 \mathrm{r} / \mathrm{min}, 25 \mathrm{r} / \mathrm{min}$ and $35 \mathrm{r} / \mathrm{min}$ respectively.

\section{Results and Discussion}

\subsection{Flow Field Distribution}

The temperature and time for precooking can be set according to the process parameters in the texture change curve of the heat processing of the shellfish, the precooking temperature will reach $100^{\circ} \mathrm{C}$ and precooking time is controlled in $60 \mathrm{~min}$ [16]. Comparing the flow field distribution of vertical axial plane at different speed. The Rotation speed reached to $15 \mathrm{r} / \mathrm{min}, 25 \mathrm{r} / \mathrm{min}$ and $35 \mathrm{r} / \mathrm{min}$.

Figure 7 shows the vertical liquid flow field at different speed. As we have seen, due to mixer, forming a cycle in the pot. The liquid flow from the liquid level along the mixer, which lash the bottom of the pot and then flow from the pot wall to the liquid level. Solid particles are driven by impact force and liquid speed, which are mixed or suspended, so that to achieve the purpose of mixer. Figure 8 (a) shows liquid phase distribution is very uneven when the rotation speed is $15 \mathrm{r} / \mathrm{min}$. As you can see, the volume fraction of the liquid phase is maximum in the upper part of the mixing pot, and the solid particles are not well suspended which influences the mixing effect. There is an inverted cone at the bottom of the blender, in which the fluid flow from bottom to top whose direction is different from the other regions, and the speed is very small [17].

Figure 7 (b) shows flow field of the vertical surface of the mixing shaft when the speed of the mixer is $25 \mathrm{r} / \mathrm{min}$. As we have seen, liquid phase distribution is uniform. The high speed area of the liquid phase is mainly concentrated in the vicinity of the mixer, so that the solid particles are not easy to precipitate.

The flow field of the vertical surface of the mixing shaft when the speed of the mixer is $35 \mathrm{r} / \mathrm{min}$ is shown in Figure 7 (c). As the rotation speed is larger, the impact force and the speed of the liquid flow are also increased. The liquid phase volume fraction is larger in the inner side of the mixer.
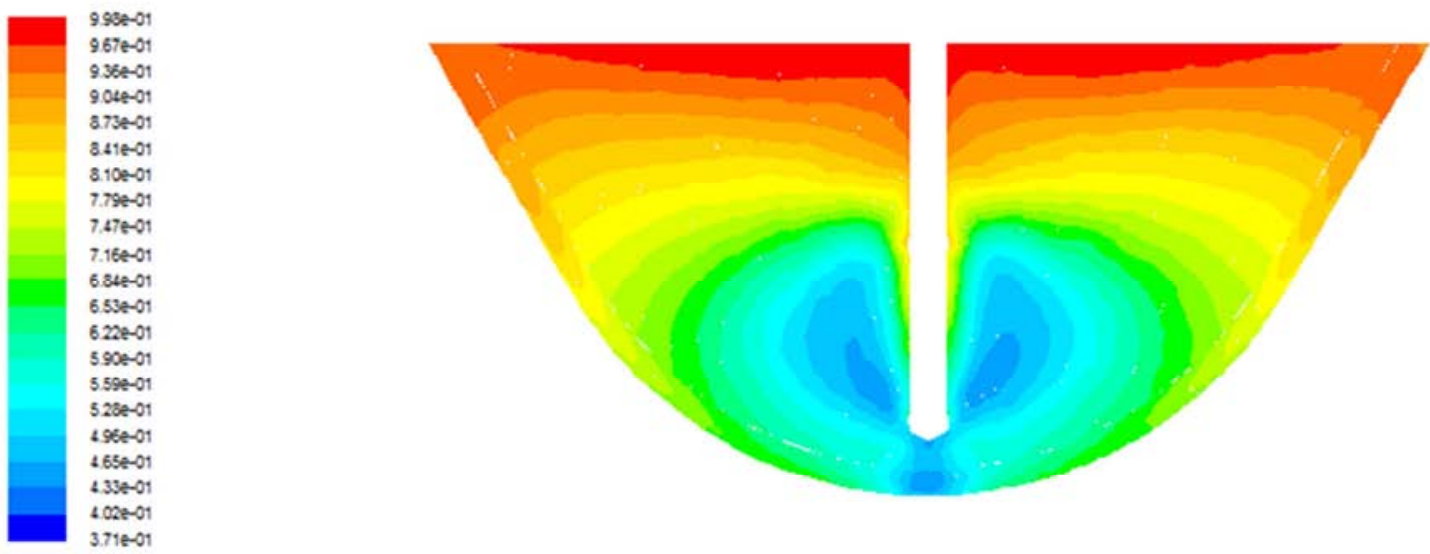

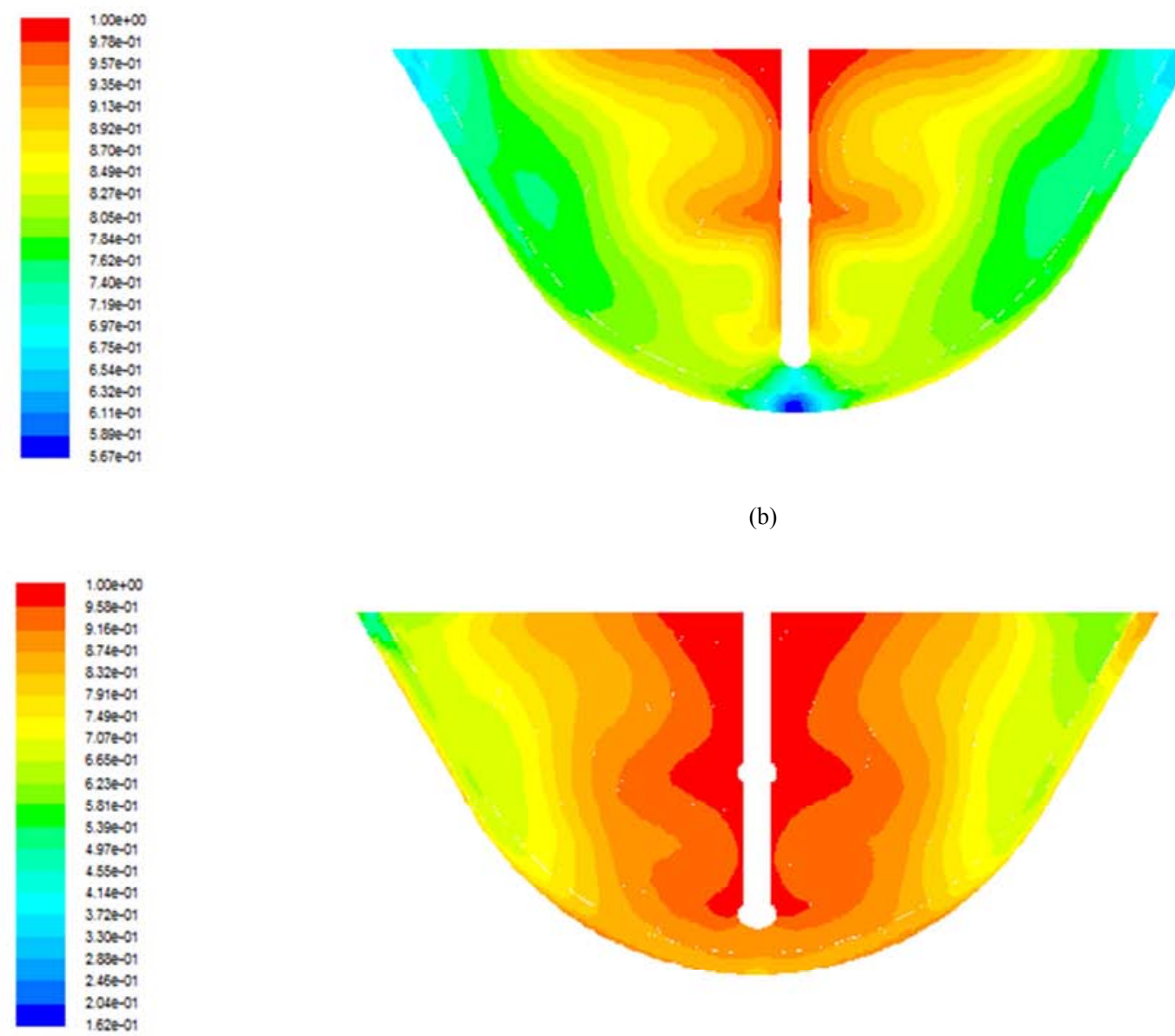

(c)

Figure 7. Comparison of vertical liquid flow field at different speeds.

\subsection{Concentration Distribution}

It is found that the flow field distribution is more uniform when the rotation speed is $25 \mathrm{r} / \mathrm{min}$. The concentration distribution for the speed of the mixer at $25 \mathrm{r} / \mathrm{min}$ is analyzed.

As shown in Figure 8 and Figure 9, solid particle volume fraction distribution are simulated when the rotation speed is $25 \mathrm{r} / \mathrm{min}$. Figure 8 shows volume fraction of solid particles in a vertical plane. Figure 9 shows the Horizontal solid particle volume fraction, in which (a) is the volume fraction of solid particles in the horizontal section of the $50 \mathrm{~mm}$ at the bottom of the pot, and (b) is the volume fraction of solid particles in the horizontal section of the $5 \mathrm{~mm}$ at the bottom of the pot. From the point of view of the distribution of solid particle volume fraction, the solid volume fraction in the bottom of the center position and the bottom wall is maximum. The volume fraction of the lower part of the mixer and the whole large circulation vortex area is the lowest.
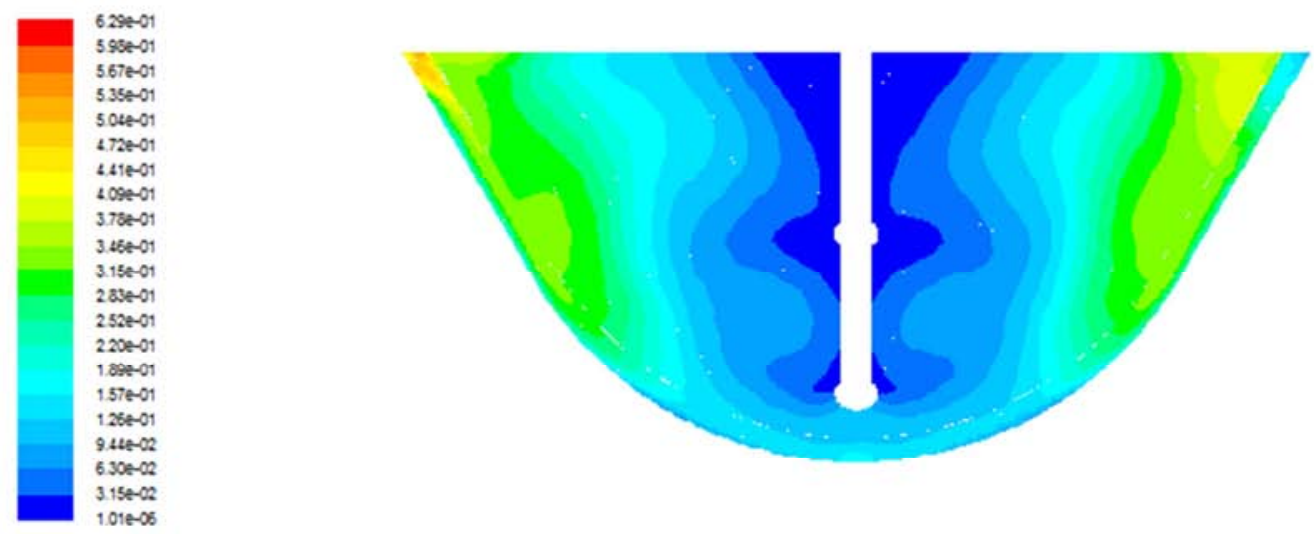

Figure 8. Volume fraction of solid particles in vertical plane. 


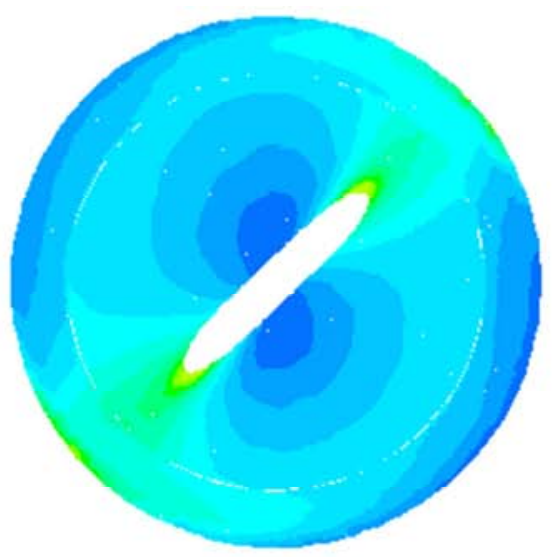

(a) Volume fraction of solid particles from the bottom $50 \mathrm{~mm}$ level

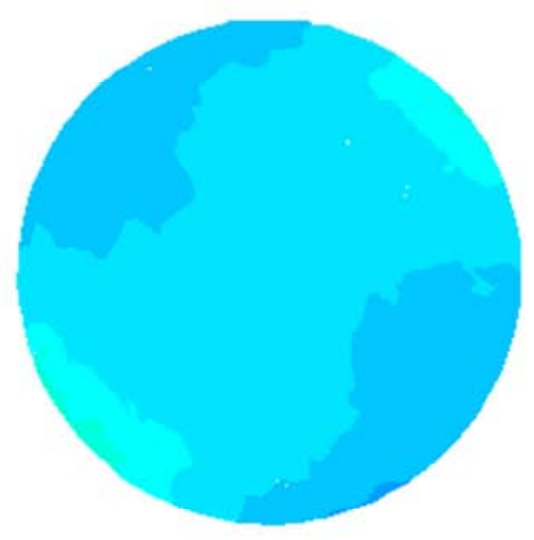

(b) Volume fraction of solid particles from the bottom $5 \mathrm{~mm}$ level

Figure 9. Horizontal solid particle volume fraction.

Analysis of the reasons for this phenomenon is that the solid particles are affected by two forces that the shear forces of the liquid flow and the gravity of the solid particles themselves in the process of mixing. The shear forces of the liquid flow makes the solid particles have a tendency to flow with the liquid. And the own gravity keeps the solid particles downward acceleration. The location of the solid particles to be deposited is the result of the combined action of the two forces. At the liquid level, the liquid flow rate is lower, and the gravity plays a leading role, so the solid particles are relatively low. The liquid flow insulating zone is formed due to the presence of the inverted cone flow pattern in the bottom of the mixer, where is high volume fraction region. There will be universal phenomenon that solid particles will be deposited at the bottom. But the bottom of the pot is curved, so that the solid particles will be greatly reduced, which is beneficial to the mixing.

\subsection{Comparison of the Textural Properties of Abalone at Different Speeds}

The tenderness and hardness of abalone were tested by TPA, which comparing with the reference value [18].

Figure 10 shows comparison of the measured value and the reference value of the tenderness and hardness of abalone at different velocities. As we have seen, the change trend of the measuring curve and the reference curve are consistent at different velocities. The measured value curve for the tenderness and hardness of abalone is more close to the reference value when the mixing speed is $25 \mathrm{r} / \mathrm{min}$, which are smaller deviations compared to the others.

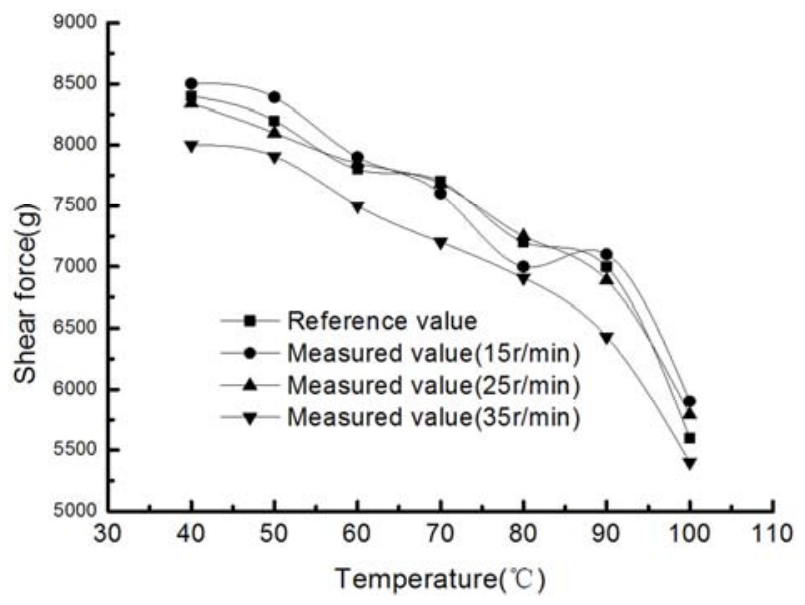

(a) Comparison of tenderness measured value and reference value curve 


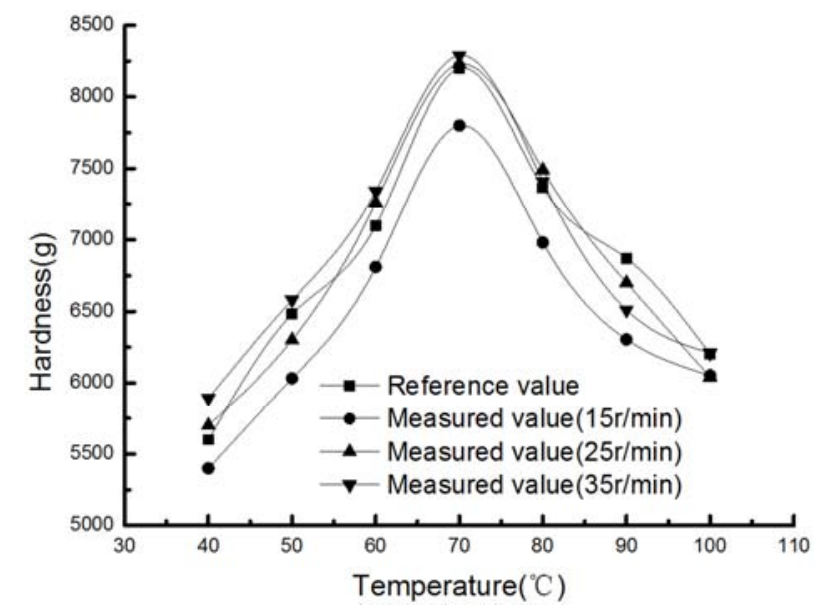

(b) Comparison of hardness measured value and reference value curve

Figure 10. Three different velocities comparison from the textural properties of abalone.

According to the detection of the textural properties for abalone that compared with the reference value. It is beneficial to the mixing when the mixing speed is $25 \mathrm{r} / \mathrm{min}$.

\section{Conclusion}

In this paper, according to the actual demand of the shellfish precooking processing machine, a new type of shellfish precooking processing machine is designed which based on the advanced processing technology. The intelligent combination unit is adopted in the elevating device, the characteristics of which is higher operating speed, lower noise, longer maintenance-free, easy to establish accuracy and excellent. The material is heated evenly by use of the tilting steam jacket kettle to achieve high thermal area and high heating efficiency.

The shape of the mixer is different from the conventional, which is tubular cross circular arc. The fluid field about the mixer is simulated at rotational velocities. The flow direction and the law of the simulation are the same as the results of the previous researchers. Moreover, the arc-shaped bottom reduced accumulation of solid particles, which is beneficial to the mixing. The results of the simulation and the design requirements are in line with.

From the whole flow pattern of the liquid flow, the liquid flow from the liquid level along the mixer, which lash the bottom of the pot and then flow from the pot wall to the liquid level. Solid particles are driven by impact force and liquid speed which are mixed or suspended, so that to achieve the purpose of mixer. From the point of view for comparison of the distribution of the flow field and the textural properties of abalone at different velocities, the mixing speed for $25 \mathrm{r} / \mathrm{min}$ is better for precooking processing.

In future study, it is necessary to comprehensively analyze the factors, which affect the uniform distribution of the mixer. And the shape of the mixer is further optimized to meet the needs of production.

\section{Acknowledgments}

The authors acknowledge the National Engineering Research Center of Seafood. This work was supported by Public Science and Technology Research Funds Projects of Ocean (No. 201505029).

\section{References}

[1] Wang, S. 2007, "Development and utilization of ancient agricultural China ancient shellfish", Ancient and Modern Agriculture, vol. 63, no. 2, pp. 22-33.

[2] Shen, J. Lin, W. Yu, W. Zhang, C. H. \& Qin, X. M. 2008, "Current situation and development prospect of Chinese shellfish processing", China Fisheries, vol. 9, no. 1, pp. 73-75.

[3] Jiang, C. J. 2012, "Main problems and Development Countermeasures of shellfish processing industry in China", Fisheries Information \& Strategy, vol. 27, no. 2, pp. 87-93.

[4] Ouyang, J. \& Shen, J. 2014, “Current situation and Prospect of application of shellfish processing equipment in China", Meat Research, vol. 28, no. 7, pp. 28-31.

[5] Goya, A. B. Bellonio, D. Bonavigna, R. \& Goya, R. G. 2012, "Quick freezing process in shellfish: Total volatile basic nitrogen and algal biotoxins values in scallops frozen on board in Argentina", Cryobiology, vol. 65, no. 3, pp. 359-360.

[6] Murchie, L. W. Cruz-Romero, M. Kerry, J. P. Linton, M. Patterson, M. F. \& Smiddy, M. 2005, "High pressure processing of shellfish: A review of microbiological and other quality aspects", Innovative Food Science \& Emerging Technologies, vol. 6, no. 3, pp. 257-270.

[7] Shang, J. H. 2013, Automatic mechanical design, Chinese Light Industry Press.

[8] Wang, C. L. Ma,, Q. Y. Li, T. \& Peng, N. 2007, "Stirring slurry pond of solid-liquid two-phase flow mixing numerical simulation", Journal of Drainage and Irrigation Machinery Engineering, vol. 25, no. 6, pp. 38-41.

[9] Du, F. L. Li, S. \& B. He, L. 2013, "Numerical simulation of solid-liquid two-phase flow in a stirred tank of $\mathrm{V}$ type propeller agitator", Manufacturing Automation, vol. 39, no. 9, pp. 3-5.

[10] Zhong, L. 2003, "Numerical simulation of solid liquid suspension in stirred tank", Beijing University of Chemical Technology, pp. 40-42.

[11] Wang, Z. S. Li, L. C. \& Huang. X. B. 2005, "CFD simulation of bottom flow field in solid liquid stirred tank," Journal of Beijing University of Chemical Technology, vol. 32, no. 4, pp. 5-9.

[12] Launder, B. E. \& Spalding, D. B. 1974, "The numerical computation of turbulent flows", Computer Methods in Applied Mechanics \& Engineering, vol. 3, no. 2, pp. 269-289.

[13] Zhu, B. W. 2013, "Research on the theory and technology of the processing of sea treasures", Science Press, pp. 18-24.

[14] Hu, X. D. Tian, Q. Qi, Z.\& Jiang, D. 2013, "Based on the sliding mesh reactor internal fluid dynamics", Pressure Vessel, vol. 23 , no. 7 , pp. 30-38. 
[15] Wang, M. H. Yang, Y. L. Gao, Z. L. Li, H. L. Zhang, J. \& Yu, J. H. 2012, "Analysis of commonly used blender in food processing", Agricultural Products Processing and Comprehensive Journal, vol. 35, no. 9, pp. 74-76.

[16] Xiao, G. H. Zhu, B. W. Dong, X. P. Chen, X. J. \& Chen, Z. 2012, "Effect of thermal processing conditions on the processing characteristic of gastropod abalone", Journal of Dalian University of technology, vol. 31, no. 1, pp. 36-38.
[17] Shen, H. P. \& Zhang, S. L. 2001, "Flow field analysis of propeller, 45 degree blade impeller and new type axial flow impeller", Journal of the University of Petroleum and Chemical Engineering, vol. 14, no. 1, pp. 55-58.

[18] Xiao, G. H. Zhu, B. W. Dong, X. P. Chen, X. J. \& Zheng, J. 2010, "Gastropod abalone in different parts of the structure characteristics and tissue structure", Food Science and Technology, vol. 12, no. 11, pp. 155-159, 2010. 